\title{
Sodium Dodecyl Sulfate Polyacrylamide Slab Gel Electrophoresis and Hydroxyethyl Cellurose Gel Capillary Electrophoresis of Luminescent Lanthanide Chelate-labeled Proteins with Time- Resolved Detection
}

\author{
Yoshinori Yamaguchi, ${ }^{* 1}$ Kimikazu Hashino, $* 2, * 3$ Masahiro Ito, ${ }^{* 4}$ Keisuke IKawa, ${ }^{* 4}$ Takuya \\ NishiOKA, ${ }^{* 2, * 4}$ and Kazuko MATSUMOTO $* 3, * 4, * 5 \dagger$ \\ *1 Consolidated Research Institute for Advanced Science and Medical Care (ASMeW), Waseda University, \\ 513 Wasedatsurumaki-cho, Shinjuku, Tokyo 162-0042, Japan \\ *2 Advanced Research Institute for Science and Engineering, Waseda University, \\ Okubo, Shinjuku, Tokyo 169-8555, Japan \\ *3 CREST, Japan Science and Technology Agency, Kawaguchi, Saitama, Japan \\ *4 Department of Chemistry, School of Science and Engineering, Waseda University, \\ Okubo, Shinjuku, Tokyo 169-8555, Japan \\ *5 Tokyo Chemical Industry Co., Ltd., 4-10-2 Nihonbashi-honcho, Chuo, Tokyo 103-0023, Japan
}

\begin{abstract}
Proteins labeled with a luminescent lanthanide chelate, $\left\{\left\{2,2^{\prime}, 2^{\prime \prime}, 2^{\prime \prime \prime}-\left\{4^{\prime}-\{[(4,6\right.\right.\right.$-dichloro-1,3,5-triazin-2yl)amino]biphenyl-4-yl $\}-2,2^{\prime}: 6^{\prime}, 2^{\prime \prime}$-terpyridine-6,6"-diyl $\}$ bis(methylenenitrilo) $\}$ tetrakis(acetato) \}europium(III) (DTBTA$\mathrm{Eu}^{3+}$ ), were analyzed with sodium dodecyl sulfate-polyacrylamide gel (SDS-PAGE) slab gel electrophoresis and hydroxyethyl cellurose gel capillary electrophoresis with a time-resolved fluorometric detector. The metal ion of the luminescent lanthanide chelate did not dissociate from the ligand during electrophoresis, and the luminescence was retained. On a slab gel, the band of DTBTA-Eu ${ }^{3+}$-labeled streptavidin was apparently less broad than that of AlexaFluor 488-labeled streptavidin. DTBTA-Eu ${ }^{3+}$ in SDS-PAGE slab gel is stable, and the gel after electrophoresis can be dried and stored for at least one year without luminescence fading. In capillary gel electrophoresis (CGE), five labeled proteins with different molecular weight were separated, and a good correlation was observed between the molecular weight and the migration time. Although the detection limits of these proteins determined in buffer solutions of the capillary electrophoresis were not better than those reported for CGE with laser-induced-detection, the detection limits of the same proteins in the present CGE system were not significantly deteriorated in serum solutions compared to those in buffer solutions, and the advantage of using time-resolved detection has been shown.
\end{abstract}

(Received November 11, 2008; Accepted January 13, 2009; Published March 10, 2009)

\section{Introduction}

Time-resolved fluorometry using lanthanide chelate (TRF-Ln) as luminescent labels has been used in many biochemical

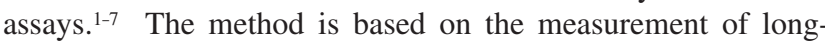
lived luminescence of a lanthanide-chelate (lifetime of several hundred $\mu$ s to more than $1 \mathrm{~ms}$ ) with an initial delay time of 100 to $400 \mu \mathrm{s}$. During the delay time, short-lived background fluorescence decay occurs, whereas the long-lived lanthanide signal remains after the delay time, and this lanthanide signal is measured after the delay time. Therefore the background fluorescence can be removed in TRF-Ln, and lanthanide-labeled target molecules are detected with high sensitivity. TRF-Ln as a detection tool has achieved many successful applications, e.g., receptor-ligand binding assay, ${ }^{8}$ immunoassay, ${ }^{9,10}$ and hybridization assay. ${ }^{9,10}$ These applications have always showed lower detection limits, compared to those of the corresponding conventional fluorometry, or many other detection methods.

$\dagger$ To whom correspondence should be addressed.

E-mail: kmatsu@yf6.so-net.ne.jp
The detection limits of TRF-Ln in immunoassay were improved by 1 to 3 orders of magnitude, ${ }^{11,12}$ and in some cases, certain proteins in serum and tissues, that had not been detected with conventional immunoassay, were successfully determined..$^{13,14}$ We have previously applied a $\beta$-diketonate- $\mathrm{Eu}^{3+}$ label for the analysis of estrogens in water samples by HPLC, and a one order of magnitude better detection limit was obtained compared to other HPLC detections. ${ }^{15}$

We have recently developed a new luminescent lanthanide chelate label, $\left\{\left\{2,2^{\prime}, 2^{\prime \prime}, 2^{\prime \prime \prime}-\left\{4^{\prime}-\{[(4,6\right.\right.\right.$-dichloro-1,3,5-triazin-2yl)amino]biphenyl-4-yl $\}-2,2^{\prime}: 6^{\prime}, 2^{\prime \prime}$-terpyridine-6, 6"-diyl \}bis(methylenenitrilo)\} tetrakis(acetato) \} europium(III) (DTBTA$\left.\mathrm{Eu}^{3+}\right)\left(\lambda_{\text {ex.max. }}=335 \mathrm{~nm}, \lambda_{\text {em.max. }}=616 \mathrm{~nm}\right),{ }^{16}$ which is stable in terms of metal dissociation and photo-degradation, and does not lose luminescence intensity appreciably in common buffers, such as EDTA, phosphate, and carboxylate. ${ }^{16,17}$ DTBTA-Eu ${ }^{3+}$ has shown a significant improvement as a luminescent lanthanide label for bio-analysis, compared to previous lanthanide labels. Most previous lanthanide labels had either luminescence intensities heavily buffer- and environmentdependent, ${ }^{16}$ or only moderately strong luminescence in some stable chelates, and thus their application in some cases had not 
satisfied the need of the assay. The luminescence of previous lanthanide chelates having not sufficiently high complex stability, decreased substantially in phosphate or carbonate buffer, possibly by substitution of some of the coordinating atoms of the chelate with buffer molecules or solvent water molecules. In our recent work, DTBTA-Eu ${ }^{3+}$ was labeled to streptavidin and used in immunoassay and DNA array analysis on a solid support to show improved detection limits compared to the conventional assay methods..$^{14,17-20}$ DTBTA-Eu ${ }^{3+}$-labeled streptavidin can be widely used in many assay formats as a versatile tag to biotin-linked proteins and nucleic acids.

Capillary gel electrophoresis is a widely used separation technique, ${ }^{21-23}$ to which application of a lanthanide chelate as a luminescent label to proteins, nucleic acids, and other biomolecules is easily conceivable, but has scarcely been reported. In the literature, capillary electrophoresis is used to study the kinetics of several complex formation of lanthanide ions with chelating ligands, ${ }^{24}$ or to separate and determine rare earth ions by using different kinetics of each ion in the electrophoresis. ${ }^{25,26}$ The use of a lanthanide chelate for biomolecules as a luminescent tag in capillary gel electrophoresis poses several obstacles to overcome that are specific to lanthanide chelates, as compared to conventional organic dye labels, i.e., the high electric voltage used in electrophoresis might cause dissociation of the positively charged metal ion from the chelate ligands. The second problem is that the mobility of the labeled proteins might be disturbed under an electric field by such a heavy label, like DTBTA-Eu ${ }^{3+}$ with a negative charge $(-1)$ as a whole, and the protein ladder commonly used to deduce the molecular weight of unknown proteins may not hold. As the third problem, heterogeneity of the labeled protein, caused by different labeling sites and different labeling numbers, might induce the broadening of a band, or a band might even be split into several components, under such highly efficient separation of capillary gel electrophoresis. From a different point of view, the high separation efficiency of electrophoresis might be able to evaluate the degree of homogeneity of a labeled protein sample.

In the present study, the performance of DTBTA-Eu ${ }^{3+}$ as a label to proteins in hydroxyethyl cellurose (HEC) gel electrophoresis was evaluated in capillary gel electrophoresis together with a sodium dodecyl sulfate-polyacrylamide gel (SDS-PAGE) slab electrophoresis to determine (1) the stability of the luminescent lanthanide chelate under an electric field when the metal-chelate is labeled to proteins, (2) the separation performance and the sensitivity improvement of time-resolved detection for several DTBTA-Eu ${ }^{3+}$ labeled proteins in the capillary SDS-PAGE system, and (3) the homogeneity of the labeled proteins observed in slab SDS-PAGE, as compared to a conventional organic dye, AlexaFluor 488. Previously, a few analyses of several lanthanide complexes by capillary electrophoresis had been reported, but in those systems the metal ion was complexed with a ligand either on the column with a free ligand added in the carrier buffer to avoid metal dissociation, ${ }^{24-29}$ or a metal ion was introduced after capillary electrophoresis in order to avoid metal dissociation during electrophoresis. ${ }^{30}$ Early in lanthanide chelate research, a Tb chelate complex with a ligand obtained from the reaction of sodium $p$-aminosalicylate with diethyltriamine pentaacetic acid (DTPA) dianhydride was reported to be a stable Tb chelate label for DNA in gel electrophoresis. ${ }^{31}$ The label was stable during electrophoresis and stable on-line monitoring of the $\mathrm{Tb}$ luminescence was possible. Although the label was stable in an electric field at elevated temperature $\left(60^{\circ} \mathrm{C}\right)$, a clear improvement of the sensitivity by time-resolved measurements was not reported. However, this Tb label was the first example showing stable migration in electrophoresis, and a second one was the $\mathrm{Eu}^{3+}$ complex of a polyaminocarboxylate ligand, which however was detected with UV absorption of the ligand. ${ }^{26}$

In the present study, DTBTA-Eu ${ }^{3+}$ having high chelate stability ( $\mathrm{p} K c a .25),{ }^{16}$ was labeled to proteins before electrophoresis, and the mobility of the labeled proteins including an antigenantibody complex, the stability of the label, and detection limits of several proteins in the time-resolved measurement were examined. A preliminary report on the use of the DTBTA-Eu ${ }^{3+}$ label for nucleic acids in capillary electrophoresis was reported, ${ }^{32}$ but its application to proteins was not evaluated. In the present study, the stability of the label, sensitivity evaluation, and application to proteins have been studied for a time-resolved fluorometry (TRF) of HEC capillary gel electrophoresis (CGE). In some cases, SDS-PAGE slab electrophoresis was used to check the homogeneity of the labeled protein and the stability of the gel after long-term storage.

\section{Experimental}

\section{Reagents and chemicals}

Chicken egg-white lysozyme $\left(M_{\mathrm{W}}=14.300\right)$, bovine milk $\beta$ lactoglobulin $\left(M_{\mathrm{W}}=18400\right)$, bovine pancreatic trypsinogen $\left(M_{\mathrm{W}}\right.$ = 24000), ovalbumin $\left(M_{\mathrm{W}}=45000\right)$, and bovine serum albumin (BSA) $\left(M_{\mathrm{W}}=66000\right)$ were purchased from Sigma-Aldrich (St. Louis, MO). Human immunoglobulin $\mathrm{G}$ (IgG) was the product of Zymed (San Francisco, CA). Streptavidin was purchased from CHEMICON International Inc. (Temecula, CA). Acylamide/bis solution (40\% acrylamide/bis solution 29:1 $(3.3 \%$ C)) was purchased from Bio-Rad Laboratories (Hercules, CA). AlexaFluor 488 was purchased from Molecular Probes Inc. (Eugene, OR). Hydroxyethyl cellulose (HEC, $\left.M_{\mathrm{W}}=90000\right)$ and $1 \mathrm{M}$ Tris-HCl buffer were purchased from Sigma (St. Louis, MO). Tris-borate-EDTA buffer (TBE; 10×) was from Bio-Rad (Hercules, CA). DTBTA-Eu ${ }^{3+}$ was synthesized as previously reported. ${ }^{16}$

\section{Apparatus}

A UV-trans illuminator (CPL20-B, COSMO BIO, Tokyo, Japan) was used for fluorescent signal detection on a slab gel. Time-resolved fluorescence image detection of a slab gel was carried out with a lab-made time-resolved fluorescence image detector, reported elsewhere. ${ }^{18}$

The lab-made capillary gel electrophoresis system with a time-resolved luminescence photon counter is shown in Fig. 1. The time-resolved luminescence photon counter was order-made by Shimadzu Co. (Kyoto, Japan). The capillary was a fusedsilica capillary with effective and total lengths of 20 and $35 \mathrm{~cm}$, respectively, and the diameters were $75 \mu \mathrm{m}$ i.d. and $365 \mu \mathrm{m}$ o.d. (Polymicro Technologies, Phoenix, AZ). The capillary was covalently coated with polyacrylamide. ${ }^{33,34}$ For excitation, a 15-W Xenon flash lamp (Hamamatsu Photonics, Hamamatsu, Japan) was used with a pulse frequency of $500 \mathrm{~Hz}$. The excitation light was focused on the capillary after passing a 350$\mathrm{nm}$ band-pass filter, and the emission light was detected after passing a 615-nm band-pass filter, with a photomultiplier tube (H8259, Hamamatsu Photonics, Japan). The excitation gate was $10 \mu \mathrm{s}$, the delay time was $100 \mu \mathrm{s}$, and the counting time was $1800 \mu$ s. Other details of the system were described previously. ${ }^{32}$

\section{Preparation of DTBTA-Eu ${ }^{3+}$-labeled proteins}

A $500-\mu l$ portion of a DTBTA-Eu ${ }^{3+}$ solution ${ }^{15}(1 \mathrm{mg} / \mathrm{ml})$ in a $100 \mathrm{mM}$ sodium bicarbonate buffer ( $\mathrm{pH}$ 9.1) was added to 500 


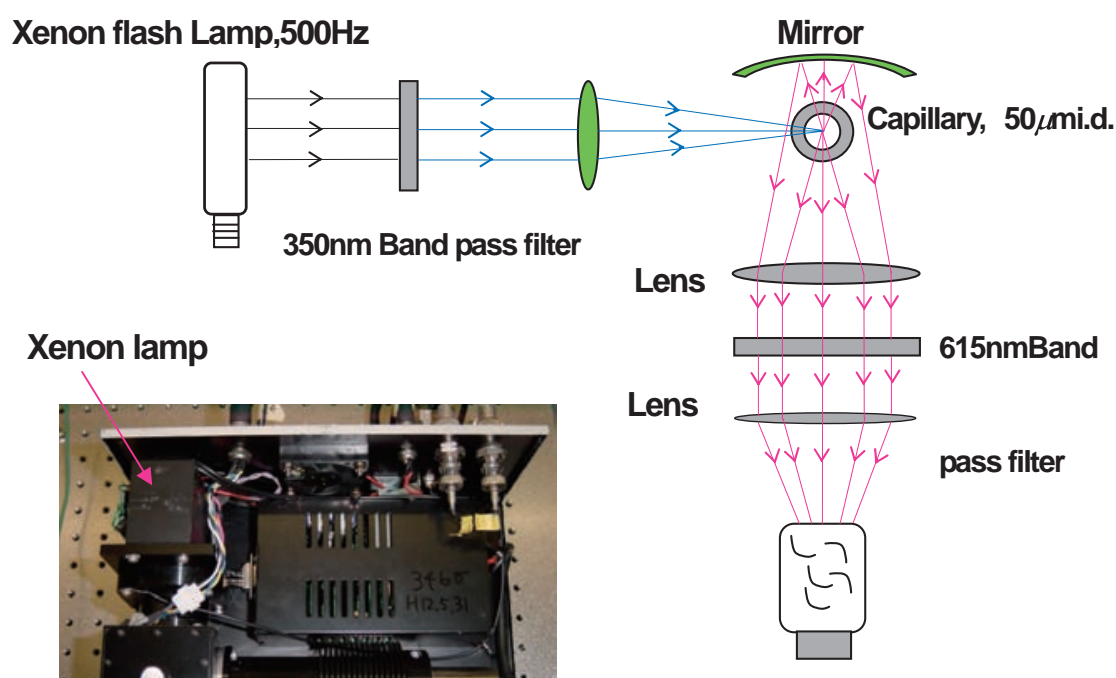

Photon Counting PMT

Capillary cell

PMT

Fig. 1 Schematic drawing and a photo of the time-resolved luminescence detector.

$\mu \mathrm{l}$ of a standard protein solution $(2 \mathrm{mg} / \mathrm{ml}$ in the same buffer). The mixture was left for $3 \mathrm{~h}$ at room temperature, and then applied on a Sephadex G-25 column to remove any unlabeled reagent. Protein fractions were separated and pooled. This labeling procedure was used for all proteins.

\section{Slab and capillary electrophoresis and detection}

A mixed solution of DTBTA-Eu ${ }^{3+}$-labeled standard proteins with different molecular weights (lysozyme, $\beta$-lactoglobulin, trypsinogen, ovalbumin, BSA, and $\mathrm{IgG}$ ) was prepared by mixing equal amounts of each protein solution and dilution with 100 $\mathrm{mM}$ sodium bicarbonate buffer $(\mathrm{pH}$ 9.1). The mixed solution and other labeled protein solutions were denatured at $95^{\circ} \mathrm{C}$ for $5 \mathrm{~min}$ in a running buffer with dithiothreitol (DTT) before electrophoresis. The SDS-PAGE of slab gel electrophoresis was carried out according to Laemmli's method. ${ }^{35}$ The polyacrylamide separation gel contained $0.375 \mathrm{M}$ Tris- $\mathrm{HCl}(\mathrm{pH}$ 8.8) and $0.1 \%$ SDS, and the running buffer was $0.025 \mathrm{M}$ Tris$\mathrm{HCl}, 0.192 \mathrm{M}$ glycine, and 0.1\% SDS (Tris/Gly/SDS) at $\mathrm{pH}$ 8.3. The sample solution contained the final concentrations of 0.0625 M Tris- $\mathrm{HCl}(\mathrm{pH}$ 6.8), 2\% SDS, 10\% glycerol, 5\% 2-mercaptoethanol, and $0.001 \%$ bromophenol blue. After electrophoresis, the gel was removed from the gel plate, and was exposed to UV-light with a UV-trans illuminator, or was observed with a time-resolved fluorometric image detector with excitation and detection wavelengths of 335 and $615 \mathrm{~nm}$, respectively. After a fluorescence observation, the gel was stained with Coomassie Brilliant Blue R 250 (CBB) staining.

Synthetic quartz tubes were used for disc gel electrophoresis. The gel after electrophoresis was directly exposed in the tube to UV-light with a UV-trans illuminator. After observing the fluorescence image, the gel was taken out from the tube and treated with $\mathrm{CBB}$.

A sieving matrix for TRF-CGE was prepared by dissolving 5 to $7.5 \%(\mathrm{w} / \mathrm{v})$ of HEC in a running buffer. The capillary was filled with a sieving polymer solution by using a syringe. The running buffer was Tris/Gly/SDS at $\mathrm{pH}$ 8.3. The applied voltage was $4000 \mathrm{~V}$ and sample solutions were injected at $50 \mathrm{~V} \mathrm{~cm}^{-1}$.
Other details of the capillary electrophoresis are described in pertinent places of Results and Discussion. Time-resolved detection was carried out with excitation and detection wavelengths of 350 and $615 \mathrm{~nm}$, respectively, with the delay time, gate time, and excitation interval being $0.1,0.8$, and $2 \mathrm{~ms}$, respectively.

\section{Results and Discussion}

\section{Slab SDS-PAGE of DTBTA-E $u^{3+-l a b e l e d ~ p r o t e i n s ~}$}

DTBTA-Eu ${ }^{3+}-$ labeled streptavidin showed a single band on SDS-slab-gel with normal fluorescence detection (Lane 2 in Fig. 2A) and CBB-staining (Lane 2 in Fig. 2B). A comparison of the two figures and the comparison of Lanes 2 and 3 in Fig. 2B show that an unlabeled streptavidin subunit was not contained in the DTBTA-Eu ${ }^{3+}$-labeled streptavidin sample. In contrast, a fluorescent image of AlexaFluor 488-labeled streptavidin showed at least four bands (Lane 1 in Fig. 2A), and a CBBstaining image of the Lane 1 sample also showed several bands (Lane 1 in Fig. 2B). These facts suggest that the DTBTA-Eu ${ }^{3+}$ labeled streptavidin is highly homogeneous as a conjugate, compared to the AlexaFluor 488-labeled streptavidin. The less homogeneity of the AlexaFluor 488-labeled protein seems to be due to inhomogeneous labeling, but the reason why the organic dye (AlexaFluor 488) and the lanthanide dye are so different in their labeling reactions was not explained by the present experiment.

Since DTBTA-Eu ${ }^{3+}$ emits long-lived fluorescence, the labeled $\mathrm{IgG}$ was detected with time-resolved fluorometry. The gel was dried on a dialysis membrane, and the fluorescence was measured with a time-resolved fluorescence image detector. The fluorescence image did not change significantly before or after fixation of the gel and after drying (data not shown).

Gels are often dried and stored for a long time after CBBstaining or Ag-staining. Therefore, the stability of the fluorescence of DTBTA-Eu ${ }^{3+}$ labeled to $\mathrm{IgG}$ on the gel was 


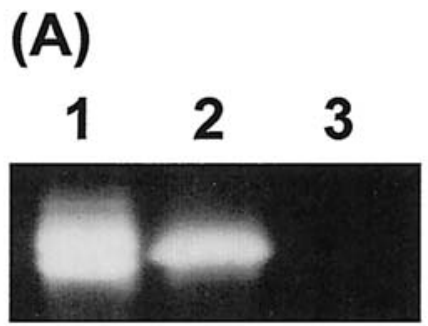

\section{(B)}

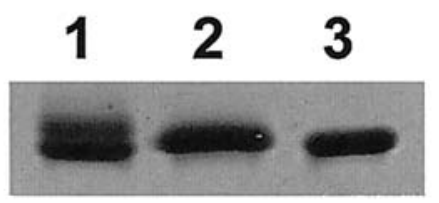

Fig. 2 Gel electrophoresis of AlexaFluor 488-labeled and DTBTA$\mathrm{Eu}^{3+}$-labeled streptavidin. After the labeling reaction, the labeledstreptavidin, which contains several fractions possibly with either different labeling number or different labeling site in the protein molecule, was electrophoresed with $17.5 \%$ polyacrylamide gel. The separated proteins were observed with a UV-trans illuminator before CBB staining (A) and after CBB staining (B). Lane 1, AlexaFluor 488-labeled streptavidin; Lane 2, DTBTA-Eu ${ }^{3+}$-labeled streptavidin; Lane 3, non-labeled streptavidin.

evaluated. No significant change was observed in the fluorescence image after 1-year storage of the dried gel (data not shown).

Disc-gel electrophoresis of labeled streptavidin and $\operatorname{Ig} G$ with time-resolved detection

Since DTBTA-Eu ${ }^{3+}\left(\lambda_{\text {ex.max. }}=335 \mathrm{~nm}, \lambda_{\text {em.max. }}=616 \mathrm{~nm}\right)$ is excited by UV light, significant background fluorescence is caused by the irradiation of UV light to tube glass. Accordingly, a conventional gel-tube made of the usual glass should not be used. Quartz tubes were used in the present experiment to avoid this problem. DTBTA-Eu ${ }^{3+}$-streptavidin was electrophoresed on disc-gel of $17.5 \%$ acrylamide, and the fluorescence signal was obtained by irradiating UV light with a trans illuminator onto the gel-tube (Fig. 3). After fluorescence observation, the gel was taken out of the tube and observed by CBB-staining. The positions of the fluorescent bands were consistent with those of TRF, as shown in Fig. 3. In the figure, impurities are more clearly observable for DTBTA-Eu ${ }^{3+}$-labeled streptavidin (column 2) than those of CBB staining (white asterisks of columns 2 and 3 in the Fig. 3). The impurity of lower molecular weight was not detected by CBB staining. These data show that TRF observation of a DTBTA-Eu ${ }^{3+}$-labeled protein on disc-gel electrophoresis is a very convenient and sensitive method to check the purity of a labeled protein. Since a TRF observation removes background fluorescence caused by the tube and gel, it is not necessary to remove the gel from the tube for CBB staining.

\section{Capillary gel electrophoresis with time-resolved detection}

Electropherograms for TRF-CGE of five DTBTA-Eu ${ }^{3+}$-labeled proteins (lysozyme, $\beta$-lactoglobulin, trypsinogen, ovalbumin, and BSA) obtained with two different HEC concentrations are shown in Fig. 4, which shows that the proteins are separated according to the protein molecular weight. The migration times of the proteins were plotted against the molecular weight, and a good linear correlation was observed (Fig. 5).

It is notable that in one case the order of the molecular weight

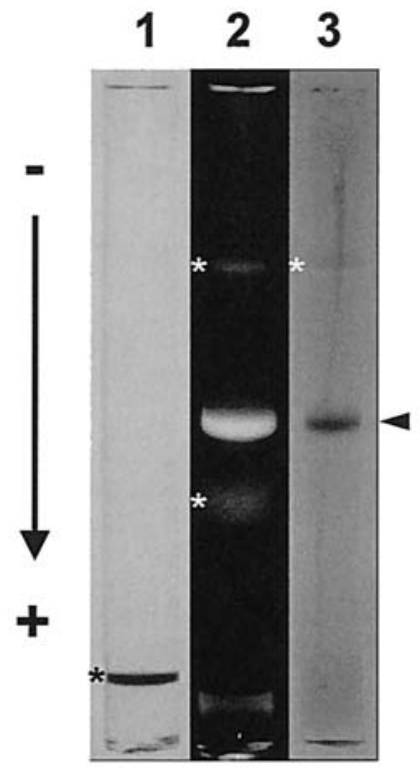

Fig. 3 Disc-gel electrophoresis of DTBTA-Eu ${ }^{3+}$-labeled streptavidin. The concentration of acrylamide gel was $17.5 \%$. Lanes 1 to 3 were observed under room light, UV light (a UV-trans illuminator), and with $\mathrm{CBB}$ staining, respectively. The arrow head indicates the DTBTA-Eu ${ }^{3+}$-labeled streptavidin. White and black asterisks are major impurities and bromophenol blue, respectively.
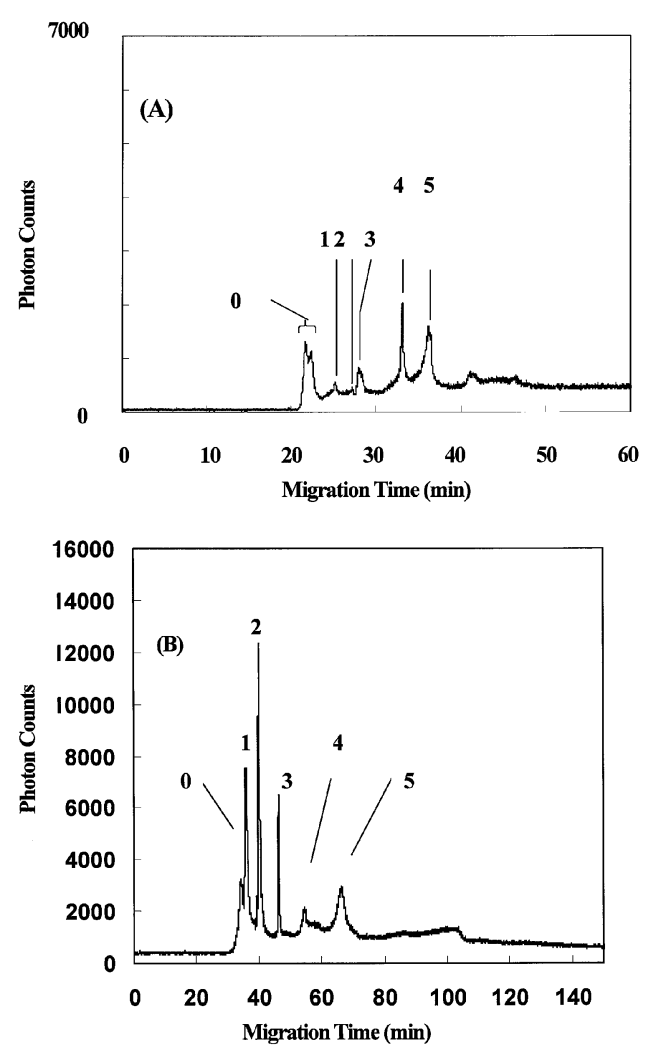

Fig. 4 Electropherograms of DTBTA-Eu ${ }^{3+}-$ labled five proteins. The protein are: 1, chicken egg-white lysozyme; 2, bovine $\beta$-lactoglobulin; 3, bovine pancreatic trypsinogen; 4, ovalbumin; 5, bovine serum albumin. Peak 0 is free DTBTA-Eu ${ }^{3+}$. (A) Applied voltage, $4000 \mathrm{~V}\left(100 \mathrm{~V} \mathrm{~cm}^{-1}\right)$; injection, $30 \mathrm{~s}\left(50 \mathrm{~V} \mathrm{~cm}^{-1}\right)$; HEC concentration, 5\%. (B) Applied voltage, $4000 \mathrm{~V}\left(100 \mathrm{~V} \mathrm{~cm}^{-1}\right)$; injection, $10 \mathrm{~s}\left(50 \mathrm{~V} \mathrm{~cm}^{-1}\right)$; HEC concentration, $7.5 \%$. 
in Fig. 5 could be reversed by full labeling of all terminal amino groups of the proteins, and a less heavy protein could become heavier by labeling, as shown in Fig. 3B. For instance, $\beta$-lactoglobulin and trypsinogen in Fig. 5 have 16 and 9 aminogroups, respectively. If all of these amino-groups were labeled, the molecular weight of $\beta$-lactoglobulin increases from 18.4 $\mathrm{kDa}$ (GenBank Accession No. CAA32835) of the non-labeled state to $33.6 \mathrm{kDa}$, and trypsinogen increases from $24 \mathrm{kDa}$ (Accession No. CAA38513) to $32.6 \mathrm{kDa}$. Therefore, the order of the molecular weights of these two proteins is reversed upon labeling, but the moblility order is retained corresponding to the non-labeled molecular weight. For such a relation, it is conceivable that not all of the amino-groups are labeled, and the order of the molecular weight is still retained after labeling. However, even so, it is notable that the mobility of the proteins is correlated with the non-labeled molecular weight. Since the proteins used in the present study were non-denatured, all amino-groups might not be located at easily accessible positions in the labeling reaction.

The split in the peak of the free DTBTA-Eu ${ }^{3+}$ in Figs. 4A and $4 \mathrm{~B}$ is due to $o-, m-$, and $p$-isomers of the DTBTA ligand, as previously reported. ${ }^{32}$ Table 1 summarizes the detection limits of the five standard proteins both in simple solution and in bovine serum solutions obtained with the present TRF-CGE system. These values are compared with those of literature values for CGE with UV absorption (UV) and laser-induced fluorescence (LIF) detections. Owing to the efficient background removal by TRF using the lanthanide chelate label, the detection limits are not worsened in serum solutions, and almost comparable detection limits are obtained both in buffer and serum solutions. In order to show the efficiency of TRF detection for background fluorescence removal, a bovine serum solution was measured with and without a delay time (Fig. 6). Due to the fluorescence from the serum components, gel materials, and capillary materials, substantially strong background fluorescence was observed for the measurement without a delay time, which, however, was removed by employing a delay time of $100 \mu \mathrm{s}$. This is a remarkable proof of the effectiveness of the TRF measurement, which explains the excellent detection limits given in Table 1, and shows that such low detection limits can be obtained without an expensive high-power laser for excitation. Figure 6 shows that serum background fluorescence contains both a flat signal and many strong peaks, which can be false signals in normal fluorescence measurements. The background level in Fig. 6 is reduced to less than $15 \%$ and no peak is observed for measurements with a 100 $\mu$ s delay time. The background level can be further reduced by elongating the delay time, but the lanthanide signal will also be reduced, and therefore the best delay time should be determined by a compromise of these two factors. Although the serum background level in LIF measurements was not found in the literature, it should be comparable to that observed without the delay time shown in Fig. 6. The background peaks in Fig. 6 should be considered to be false peaks, and separation of the analyte peaks from the false peaks becomes a major obstacle in the non-time-resolved fluorometric trace analysis of serum samples. In contrast, TRF-CGE can be an easy and superb tool for the analysis of such samples.

\section{Conclusions}

The performance of standard proteins labeled with a new $\mathrm{Eu}^{3+}$ chelate reagent in SDS-PAGE slab gel electrophoresis and HEC gel capillary electrophoresis was studied. Although proteins were labeled with a heavy metal chelate, they were separated according to their molecular weight, and a good correlation was observed between the molecular weight and the migration time.

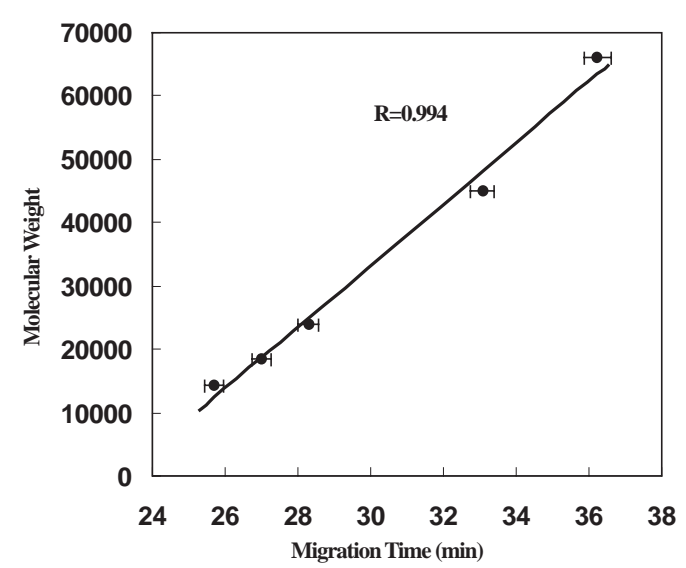

Fig. 5 Correlation of the migration time with the molecular weight observed for Fig. 4(A).

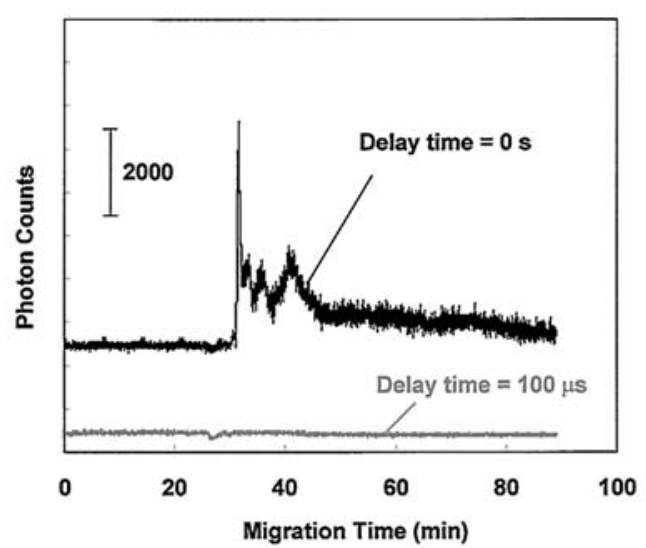

Fig. 6 Effect of the delay time in the TRF measurement upon removal of any background fluorescence.

Table 1 Comparison of the detection limits (nM) of several proteins in SDS-PAGE capillary electrophoresis

\begin{tabular}{lccccc}
\hline & Lysozyme & $\beta$-Lactoglobulin & Typsinogen & Ovalbumin & BSA \\
\hline UV absorption $^{36}$ & N.A. & 820 & 625 & 330 & 220 \\
LIF $^{37}$ & N.A. & N.A. & N.A. & 8 & 4 \\
LIF $^{38}$ & N.A. & 0.16 & N.A. & 0.16 & 0.09 \\
TRF-CGE $^{\text {a }}$ (buffer solution) & 4 & 5 & 1 & 1 & 0.5 \\
TRF-CGE (serum solution) & 2 & 1.5 & 1 & 1.5 & 1 \\
\hline
\end{tabular}

a. The detection limits were determined as $S / N=3$ in the present study. N.A. means "not available in the literature". 
The labeled proteins were proved to be more homogeneous, as observed in SDS-PAGE, compared with the same proteins labeled with AlexaFluor 488. Although the detection limits of several standard proteins in buffer solutions for the present SDSPAGE CGE are not as low as those reported for LIF-CGE, those of the proteins in serum in the present system shown in Table 1 are considerably low, and show that the time-resolved measurement is effective for sample solutions with a complex matrix, like serum.

\section{Acknowledgements}

The present work was supported by grants from the Japan Science and Technology Agency (Core Research for Evolutional Science and Technology (CREST) project).

\section{References}

1. I. Hemmilä, Clin. Chem., 1985, 31, 359.

2. E. P. Diamandis and T. K. Christopoulos, Anal. Chem., 1990, 62, 1149A.

3. H. Kimura, M. Mukaida, G. Wang, J. Yuan, and K. Matsumoto, Forensic Sci. Int., 2000, 113, 345.

4. P. Hurskainen, P. Dahlen, I. Ylikoski, M. Kwiatkowski, H. Siitari, and T. Lövgren, Nucleic Acids Res., 1991, 19, 1057.

5. S. Sueda, J. Yuan, and K. Matsumoto, Bioconjugate Chem., 2000, 11, 827.

6. J. L. Su, J. Fornwald, P. Rivers, S. Goldsworthy, N. A. Looney, J. Hanvey, C. Plumpton, J. Parham, M. Romanos, T. A. Kost, and F. C. Kull Jr., J. Immunol. Methods, 2004, 291, 123.

7. K. Matsmoto and J. Yuan, "Metal Ions in Biological Systems", ed. A. Sigel and H. Sigel, 2003, Vol. 40, Chap. 6, Marcel Dekker, New York, 191.

8. H. L. Handl and R. J. Gillies, Life Sci., 2005, 77, 361.

9. I. Hemmilä and V. Laitala, J. Fluores., 2005, 15, 529.

10. G. R. Motson, J. S. Fleming, and S. Brooker, Adv. Inorg. Chem., 2004, 55, 361.

11. J. Yuan, K. Matsumoto, and H. Kimura, Anal. Chem., 1998, $70,596$.

12. J. Yuan, G. Wang, K. Majima, and K. Matsumoto, Anal. Chem., 2001, 73, 1869.

13. H. Kimura, M. Mukaida, K. Kuwabara, T. Ito, K. Hashino, K. Uchida, K. Matsumoto, and K. Yoshida, Free Radical Biol. Med., 2006, 41, 973.

14. M. Ikegawa, J. Yuan, K. Matsumoto, S, Hermann, I. Iwamoto, T. Nakamura, S. Matsushita, T. Kimura, T. Honjo, and K. Tashiro, AIDS Res. Hum. Retrov., 2001, 17, 587.

15. K. Matsumoto, Y. Tsukahara, T. Uemura, K. Tsunoda, H. Kume, S. Kawasaki, J. Tadano, and T. Matsuya, J. Chromatogr., B, 2002, 773, 135.

16. T. Nishioka, J. Yuan, Y. Yamamoto, K. Sumitomo, Z. Wang, K. Hashino, C. Hosoya, K. Ikawa, G. Wang, and K. Matsumoto, Inorg. Chem., 2006, 45, 4088.

17. K. Hashino, M. Ito, K. Ikawa, C. Hosoya, T. Nishioka, and K. Matsumoto, Anal. Biochem., 2006, 355, 278.

18. K. Hashino, K. Ikawa, M. Ito, C. Hosoya, T. Nishioka, M. Makiuchi, and K. Matsumoto, Anal. Biochem., 2007, 364, 89.

19. H. Kimura, M. Mukaida, M. Watanabe, K. Hashino, T. Nishioka, Y. Tomino, K. Yoshida, and K. Matsumoto, Anal. Biochem., 2008, 372, 119.

20. M.-J. Xie, K. Fukui, M. Horie, Y. Sakihama, K. Hashino, H. Kimura, and K. Matsumoto, Anal. Biochem., 2008, 374, 278.

21. D. Baker, "Capillary Electrophoresis", 1995, Wiley, New York, Chichester.

22. S.-C. Wang and M. Morris, Anal. Sci., 2001, 17, i173.

23. S. Hu, L. Zhang, and N. J. Dovichi, J. Chromatogr., A, 2001, 924, 369.

24. X. Zhu and S. Z. Lever, Electrophoresis, 2002, 23, 1348.

25. C. Vogt and S. Conradi, Anal. Chim. Acta, 1994, 294, 145.

26. S. Saito and H. Hoshino, Anal. Bioanal. Chem., 2004, 378, 1644.

27. M. Fani, S. Vranjeo, S. C. Archimandritis, S. Potamianos, S. Xanthopoulos, P. Bouziotic, and A. D. Varvarigou, Appl. Radiat. Isot., 2002, 57, 665.

28. K. J. Franz, M. Nitz, and B. Imperiali, ChemBioChem, 2003, 4, 265.

29. R. Kautenburger, K. Nowotka, and H. P. Beck, Anal. Bioanal. Chem., 2006, 384, 1416.

30. R. Zhu and W. T. Kok, Anal. Chem., 1997, 69, 4010.

31. S. R. Saavedra and E. G. Piccozza, Analyst, 1989, 114, 835.

32. K. Sumitomo, T. Ito, M. Sasaki, and Y. Yamaguchi, Chromatographia, 2008, 67, 715.

33. S. Hjerten, J. Chromatogr., 1985, 347, 191.

34. D. Schmalzing, C. A. Piggee, F. Foret, E. Carrilho, and B. J. Karger, J. Chromatogr., A, 1993, 652, 149.

35. U. K. Laemmli, Nature, 1970, 227, 680.

36. K. Ganzler, K. S. Greve, A. S. Cohen, B. L. Karger, A. Guttman, and N. C. Cooke, Anal. Chem., 1992, 64, 2665.

37. S. Hu, L. Zhang, L. M. Cook, E. J. Carpenter, and N. J. Dovichi, J. Chromatogr., A, 2000, 894, 291.

38. S. Hu, L. Zhang, L. M. Cook, and N. J. Dovichi, Electrophoresis, 2001, 22, 3677. 